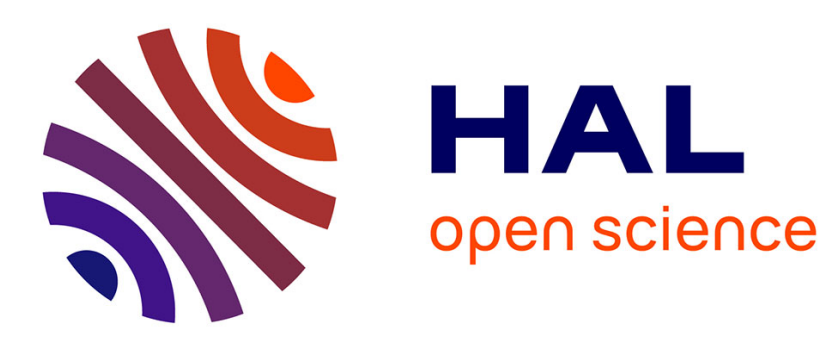

\title{
Computer and Computing Technologies in Agriculture $\mathrm{XI}$
}

\author{
Daoliang Li, Chunjiang Zhao
}

\section{To cite this version:}

Daoliang Li, Chunjiang Zhao. Computer and Computing Technologies in Agriculture XI: 11th IFIP WG 5.14 International Conference, CCTA 2017, Jilin, China, August 12-15, 2017, Proceedings, Part II. Springer International Publishing, AICT-546, 2019, IFIP Advances in Information and Communication Technology, 978-3-030-06178-4. 10.1007/978-3-030-06179-1 . hal-02111509

\section{HAL Id: hal-02111509 \\ https://hal.inria.fr/hal-02111509}

Submitted on 26 Apr 2019

HAL is a multi-disciplinary open access archive for the deposit and dissemination of scientific research documents, whether they are published or not. The documents may come from teaching and research institutions in France or abroad, or from public or private research centers.
L'archive ouverte pluridisciplinaire HAL, est destinée au dépôt et à la diffusion de documents scientifiques de niveau recherche, publiés ou non, émanant des établissements d'enseignement et de recherche français ou étrangers, des laboratoires publics ou privés. 


\section{IFIP Advances in Information and Communication Technology}

\section{Editor-in-Chief}

Kai Rannenberg, Goethe University Frankfurt, Germany

\section{Editorial Board}

TC 1 - Foundations of Computer Science

Jacques Sakarovitch, Télécom ParisTech, France

TC 2 - Software: Theory and Practice

Michael Goedicke, University of Duisburg-Essen, Germany

TC 3 - Education

Arthur Tatnall, Victoria University, Melbourne, Australia

TC 5 - Information Technology Applications

Erich J. Neuhold, University of Vienna, Austria

TC 6 - Communication Systems

Aiko Pras, University of Twente, Enschede, The Netherlands

TC 7 - System Modeling and Optimization

Fredi Tröltzsch, TU Berlin, Germany

TC 8 - Information Systems

Jan Pries-Heje, Roskilde University, Denmark

TC 9 - ICT and Society

David Kreps, University of Salford, Greater Manchester, UK

TC 10 - Computer Systems Technology

Ricardo Reis, Federal University of Rio Grande do Sul, Porto Alegre, Brazil

TC 11 - Security and Privacy Protection in Information Processing Systems

Steven Furnell, Plymouth University, UK

TC 12 - Artificial Intelligence

Ulrich Furbach, University of Koblenz-Landau, Germany

TC 13 - Human-Computer Interaction

Marco Winckler, University Paul Sabatier, Toulouse, France

TC 14 - Entertainment Computing

Matthias Rauterberg, Eindhoven University of Technology, The Netherlands 


\section{IFIP - The International Federation for Information Processing}

IFIP was founded in 1960 under the auspices of UNESCO, following the first World Computer Congress held in Paris the previous year. A federation for societies working in information processing, IFIP's aim is two-fold: to support information processing in the countries of its members and to encourage technology transfer to developing nations. As its mission statement clearly states:

IFIP is the global non-profit federation of societies of ICT professionals that aims at achieving a worldwide professional and socially responsible development and application of information and communication technologies.

IFIP is a non-profit-making organization, run almost solely by 2500 volunteers. It operates through a number of technical committees and working groups, which organize events and publications. IFIP's events range from large international open conferences to working conferences and local seminars.

The flagship event is the IFIP World Computer Congress, at which both invited and contributed papers are presented. Contributed papers are rigorously refereed and the rejection rate is high.

As with the Congress, participation in the open conferences is open to all and papers may be invited or submitted. Again, submitted papers are stringently refereed.

The working conferences are structured differently. They are usually run by a working group and attendance is generally smaller and occasionally by invitation only. Their purpose is to create an atmosphere conducive to innovation and development. Refereeing is also rigorous and papers are subjected to extensive group discussion.

Publications arising from IFIP events vary. The papers presented at the IFIP World Computer Congress and at open conferences are published as conference proceedings, while the results of the working conferences are often published as collections of selected and edited papers.

IFIP distinguishes three types of institutional membership: Country Representative Members, Members at Large, and Associate Members. The type of organization that can apply for membership is a wide variety and includes national or international societies of individual computer scientists/ICT professionals, associations or federations of such societies, government institutions/government related organizations, national or international research institutes or consortia, universities, academies of sciences, companies, national or international associations or federations of companies.

More information about this series at http://www.springer.com/series/6102 
Daoliang Li · Chunjiang Zhao (Eds.)

\section{Computer and \\ Computing Technologies in Agriculture XI}

11th IFIP WG 5.14 International Conference, CCTA 2017 Jilin, China, August 12-15, 2017

Proceedings, Part II 


\section{Editors}

Daoliang Li

China Agricultural University (CAU)

Beijing, China
Chunjiang Zhao

National Research Center of Intelligent

Equipment for Agriculture (NRCIEA)

Beijing, China
ISSN 1868-4238 ISSN 1868-422X (electronic)
IFIP Advances in Information and Communication Technology
ISBN 978-3-030-06178-4
https://doi.org/10.1007/978-3-030-06179-1

Library of Congress Control Number: 2018965408

(C) IFIP International Federation for Information Processing 2019

This work is subject to copyright. All rights are reserved by the Publisher, whether the whole or part of the material is concerned, specifically the rights of translation, reprinting, reuse of illustrations, recitation, broadcasting, reproduction on microfilms or in any other physical way, and transmission or information storage and retrieval, electronic adaptation, computer software, or by similar or dissimilar methodology now known or hereafter developed.

The use of general descriptive names, registered names, trademarks, service marks, etc. in this publication does not imply, even in the absence of a specific statement, that such names are exempt from the relevant protective laws and regulations and therefore free for general use.

The publisher, the authors, and the editors are safe to assume that the advice and information in this book are believed to be true and accurate at the date of publication. Neither the publisher nor the authors or the editors give a warranty, express or implied, with respect to the material contained herein or for any errors or omissions that may have been made. The publisher remains neutral with regard to jurisdictional claims in published maps and institutional affiliations.

This Springer imprint is published by the registered company Springer Nature Switzerland AG The registered company address is: Gewerbestrasse 11, 6330 Cham, Switzerland 


\section{Preface}

Agricultural information technology has become an important means for developing modern agriculture. In order to promote the academic exchange and cooperation of ICT in Agriculture, from 2000 to 2016, the National Engineering Research Center for Information Technology in Agriculture (NERCITA), China Agricultural University (CAU), and related organizations hosted eight events in the International Symposium on Intelligent Information Technology in Agriculture (ISIITA) series and ten events in the International Conference on Computer and Computing Technologies in Agriculture (CCTA) series, which provided a platform for the exchange of information on and ICT in agriculture between global scholars.

Since 2015, the Internet of Things, big data, robots, and precision farming have entered a stage of agricultural applications. Artificial intelligence (AI) has gradually penetrated the agriculture field and promoted the development of intelligent agriculture, which is characterized by "information perception, quantitative decision-making, intelligent control, precision input, and personalized services." To exchange experiences and share the state of the art as well as successful application cases of ICT in intelligent agriculture, ISIITA and CCTA were merged into the International Conference on Intelligent Agriculture (ICIA). The ICIA will be held every two years (odd year), and the co-sponsors and conference venue are also selected according to applications.

ICIA 2017 focused on four topics: Internet of Things and Big Data in Agriculture, Precision Agriculture and Agricultural Robot, Agricultural Information Services, and Animal and Plant Phenotyping for Agriculture. The newest theories, viewpoints, technologies, products, and applications were extensively presented. Practical experiences in innovation and applications in intelligent agriculture were shared among experts from different countries.

We selected the 100 best papers among the 282 papers submitted to CCTA 2017 for these proceedings. All papers underwent two reviews by members who are from the Special Interest Group on Advanced Information Processing in Agriculture (AIPA), IFIP. In these proceedings, creative thoughts and inspiration can be discovered, discussed, and disseminated. It is always exciting to have experts, professional, and scholars with creative contributions getting together to share inspiring ideas and to accomplish great developments in the field.

I would like to express my sincere thanks to all authors who submitted research papers to the conference. Finally, I would also like to express my sincere thanks to all speakers, session chairs, and attendees, both national and international, for their active participation and support of this conference. 


\section{Organization}

\section{Organizers}

China National Engineering Research Center for Information Technology in Agriculture (NERCITA), China

China Agricultural University (CAU), China

China National Engineering Research Center of Intelligent Equipment for Agriculture (NERCIEA), China

Branch of Agricultural Information Processing, International Federation for Information Processing (IFIP TC5.14)

Chinese Association of Artificial Intelligence (CAAI), China

Chinese Society of Agricultural Engineering (CSAE), China

Chinese Society for Agricultural Machinery (CSAM), China

Informatization Branch, China Agricultural Mechanization Association (CAMA), China

Committee of Information Technology, China Agro-technological Extension Association (CAEA), China

Technology Innovation Strategic Alliance for Agricultural Internet of Things Industry (TISA-AITI), Beijing, China

Beijing Society for Information Technology in Agriculture (BSITA), China

Key Laboratory of Information Technology in Agriculture, Ministry of Agriculture, China

Sino-US Cooperative Technology Center of Agricultural Aviation, China

Beijing Key Laboratory of Digital Plant, China

\section{Sponsors}

Department of Science, Technology and Education, Ministry of Agriculture (MOA), China

Department of Market and Economic Information, Ministry of Agriculture (MOA), China

Department of International Cooperation, Ministry of Science and Technology (MOST), China

Jilin Provincial Agricultural Commission, China

Jilin Provincial Department of Science and Technology, China

Changchun Municipal Government, China

Beijing Association for Science and Technology, China

Beijing Academy of Agriculture and Forestry Sciences, China 


\section{Organizing Committee}

Chunjiang Zhao

Nick Sigrimis

Yanbo Huang

Youhong Sun

Zhang Yuejie
China National Engineering Research Center for Information Technology in Agriculture, China

Agricultural University of Athens, Greece

Crop Production Systems Research, USDA-ARS

Jilin University, China

Jilin Agricultural University, China

\section{Academic Committee}

Maohua Wang

Jiulin Sun

Deyi Li

Xiwen Luo

Xuegeng Chen

$\mathrm{Yu} \mathrm{Li}$

Luquan Ren

Fangquan Mei

Chunjiang Zhao

Daoliang Li

Xinting Yang

Liping Chen

Yong $\mathrm{He}$

Yan Zhu

Dongjian $\mathrm{He}$

Chengliang Liu

Hanping Mao
China Agricultural University (CAU) and Chinese Academy of Engineering, China

Institute of Geographic Sciences and Natural Resources Research, Chinese Academy of Sciences and Chinese Academy of Engineering, China

61st Institute of the General Staff Headquarters of Chinese People's Liberation Army and Chinese Academy of Engineering, China

South China Agricultural University, Chinese Academy of Engineering, China

Institute of Agricultural Machinery, Xinjiang Academy of Agricultural and Reclamation Science and Chinese Academy of Engineering, China

Jilin Agricultural University, Chinese Academy of Engineering, China

Jilin University, Chinese Academy of Sciences, China

Agricultural Information Institute, Chinese Academy of Agricultural Sciences (CAAS), China

China National Engineering Research Center for Information Technology in Agriculture, China

College of Information and Electrical Engineering, China Agricultural University, China

China National Engineering Research Center for Information Technology in Agriculture, China

China National Engineering Research Center of Intelligent Equipment for Agriculture, China

College of Biosystems Engineering and Food Science, Zhejiang University, China

College of Agriculture, Nanjing Agricultural University, China

College of Mechanical and Electronic Engineering, Northwest A\&F University, China

Shanghai Jiao Tong University, China

Institute of Agricultural Engineering, Jiangsu University, China 
Haiye Yu

Shijun Li

Guifen Chen

Nick Sigrimis

Kuanchong Ting

Manuel Berenguel

Jose Fernando

Bienvenido

Rajiv Khosla

Zhang Qin

Yanbo Huang

Wesley Clint

Hoffmann

Jens Leon

Thomas

Rauschenbach

Daniela Baganz

Sun-Ok Chung

Naoshi Kondo

Noboru Noguchi

Ning Wang

Naiqian Zhang

Frederic Baret
College of Biological and Agricultural Engineering, Jilin University, China

College of Information Technology, Jilin Agricultural University, China

College of Information Technology, Jilin Agricultural

University, China

Agricultural University of Athens, Greece

Zhejiang University, China/University of Illinois at Urbana-Champaign, USA

University of Almeria, Spain

University of Almeria, Spain

Colorado State University, USA

Washington State University, USA

Crop Production Systems Research, USDA-ARS

Aerial Application Technology Group, USDA-ARS

University of Bonn, Germany

Fraunhofer-IOSB, Advanced Systems Technology Branch (AST), Germany

Leibniz Institute of Freshwater Ecology and Inland Fisheries, IGB, Germany

Chungnam National University, Republic of Korea, Korea

Kyoto University, Japan

Hokkaido University, Japan

Oklahoma State University, USA

Kansas State University, USA

French National Institute for Agricultural Research, France

\section{Additional Reviewers}

Chunjiang Zhao

Daoliang Li

Xinting Yang

Yong $\mathrm{He}$

Chengliang Liu

Zhenbo Li

Yingyi Chen

Yaoguang Wei
China National Engineering Research Center for Information Technology in Agriculture, China

College of Information and Electrical Engineering, China Agricultural University, China

China National Engineering Research Center for Information Technology in Agriculture, China

College of Biosystems Engineering and Food Science, Zhejiang University, China

Shanghai Jiao Tong University, China

College of Information and Electrical Engineering, China Agricultural University, China

College of Information and Electrical Engineering, China Agricultural University, China

College of Information and Electrical Engineering, China Agricultural University, China 
Qingling Duan

Longqing Sun

Chunhong Liu

Weizhong Yang
College of Information and Electrical Engineering, China Agricultural University, China

College of Information and Electrical Engineering, China Agricultural University, China

College of Information and Electrical Engineering, China Agricultural University, China

College of Information and Electrical Engineering, China Agricultural University, China

\title{
Secretary General
}

\author{
Xiaohong Du \\ $\mathrm{Xia} \mathrm{Li}$ \\ Ming Li \\ Hongru Wang \\ Han Zhang
}




\section{Contents - Part II}

Research and Application of Spark Platform on Big Data Processing in Intelligent Agriculture of Jilin Province . . . . . . . . . . . . . . . . . .

Siwei Fu, Guifen Chen, Shan Zhao, and Enze Xiao

Summary of Agricultural Drought Monitoring by Remote Sensing at Home and Abroad . . . . . . . . . . . . . . . . . . . .

Meng Wang, Tao Liu, Shouzhen Ling, Xueyan Sui, Huimin Yao, and Xuehui Hou

Research and Application of 3D Visualization Plug-in Integration with ArcGIS

Yinglun Li, Guifen Chen, and Dongxue Wang

Growth and Spectral Characteristics of Grassland in Response to Different Soil Textures. . . . . . . . . . . . . . . . . . . . . . . . .

Xiaochun Zhong, Junchan Wang, Liu Tao, Chengming Sun, Zhemin Li, and Shengping Liu

Soil Moisture Estimation by Combining L-Band Brightness Temperature and Vegetation Related Information. . . . . . . . . . . . . . . . . . . . .

Yuanyuan Fu, Chunjiang Zhao, Guijun Yang, and Haikuan Feng

Study on Precision Fertilization Model Based on Fusion Algorithm of Cluster and RBF Neural Network . . . . . . . . . . . . . . . . . . . . . . .

Shan Zhao, Guifen Chen, Siwei Fu, and Enze Xiao

Study on Three-Dimensional Data Acquisition of Crop Grains . . . . . . . . . .

Zetao Yu, Weiliang Wen, Xinyu Guo, and Xianju Lu

An Agricultural Habitat Information Acquisition and Remote Intelligent

Decision System Based on the Internet of Things . . . . . . . . . . . . . . .

Ze Lin Hu, Yi Gao, Miao Li, Hua Long Li, Xuan Jiang Yang, and Zhi Run Ma

Comprehensive Evaluation of Soil Fertility in Yanzhou District Based on Principal Component Analysis . . . . . . . . . . . . . . . . . . .

Qiuting Zhang and Xia Geng

Advances in Monitoring Soil Nutrients by Near Infrared Spectroscopy . . . . . 
Microwave Mixing Technique for Nondestructive Measurement of Moisture Content of Particulate Agricultural Products . . . . . . . . . . . . .

Chenxiao Li, Yanlei Xu, He Gong, Yuanyuan Liu, and Qian Song

China's Wine Import Industry: An Economic Analysis

of Influencing Trade Factors.

Yu Hu, Wei Ma, Ruimei Wang, Huan Song, Weisong Mu, Dong Tian, and Jianying Feng

Three - Dimensional Visualization of Soil Nutrient Evolution in Maize Precision Operation Area Based on ArcGIS . . . . . . . . . . . . . . . . . .

Enze Xiao, Guifen Chen, Shan Zhao, and Siwei Fu

Characteristics of the Warming Trend During Winter Wheat Growing

Seasons in Jiangsu Province of China . . . . . . . . . . . . . . . . .

Xiangying Xu, Xinkai Zhu, Wenshan Guo, Chunyan Li, and Jinfeng Ding

Estimation of Leaf Nitrogen Concentration of Winter Wheat Using

UAV-Based RGB Imagery . . . . . . . . . . . . . . . . . . . . . . . .

Qinglin Niu, Haikuan Feng, Changchun Li, Guijun Yang, Yuanyuan Fu, Zhenhai Li, and Haojie Pei

New NNI Model in Winter Wheat Based on Hyperspectral Index . . . . . . . . .

Wang Jianwen, Li Zhenhai, Xu Xingang, Zhu Hongchun, Feng Haikuan, Liu Chang, Gan Ping, and Xu Xiaobin

Fruit Trees 3D Data Acquisition and Reconstruction Based on Multi-source ...........................

Sheng Wu, Boxiang Xiao, Weiliang Wen, Xinyu Guo, and Long Liu

Comparison of Remote Sensing Estimation Methods for Winter Wheat Leaf

Nitrogen Content . . . . . . . . . . . . . . . . . . . . .

Chunlan Zhang, Fuquan Tang, Heli Li, Guijun Yang, Haikuan Feng, and Chang Liu

Design and Implementation of the Wheat Population Nutrition

Detection System ..........................

Lei Shi, Qiguo Duan, Mingyang Xiong, Juanjuan Zhang, Lihong Song, and Xinming $\mathrm{Ma}$

Hyperspectral Estimation Methods for Chlorophyll Content of Apple Based on Random Forest. . . . . . . . . . . . . . . . . . . . . . . . . . . . . . .

Haojie Pei, Changchun Li, Haikuan Feng, Guijun Yang, Mingxing Liu, and Zhichao $\mathrm{Wu}$

Improving Design of a PVDF Grain Loss Sensor for Combine Harvester . . . . Liming Zhou, Yanwei Yuan, Junning Zhang, and Kang Niu 
Maize Precision Farming Parallel Management Technology

and Its Application in Northeast China . . . . . . . . . . . . . . .

Хianju Lu, Хіпуи Guo, Jiangchuan Fan, Sheng Luo, Yufa Song,

and Chunwei $\mathrm{Li}$

Research on Vegetation Ecologic Quality Index of Rocky Desertification in Karst Area of Guangxi Province Based on NPP and Fractional Vegetation Cover Since 2000 . . . . . . . . . . . . . . . . . . . . . . . . . .

Xin Yang, Haihong Huang, Shuan Qian, and Hao Yan

Estimation of Leaf Nitrogen Content of Winter Wheat Based on Akaike's Information Criterion. . . . . . . . . . . . . . . . . . . .

Haojie Pei, Haikuan Feng, Fuqin Yang, Zhenhai Li, Guijun Yang, and Qinglin Niu

Monitoring of Winter Wheat Biomass Using UAV Hyperspectral

Texture Features . . . . . . . . . . . . . . . . . . . . . . . . . . .

Chang Liu, Guijun Yang, Zhenhai Li, Fuquan Tang, Haikuan Feng, Jianwen Wang, Chunlan Zhang, and Liyan Zhang

Reconstruction and Body Size Detection of 3D Sheep Body Model Based on Point Cloud Data . . . . . . . . . . . . . . . . . . . . . . . . .

Yanqing Zhou, Heru Xue, Chunlan Wang, Xinhua Jiang, Xiaojing Gao, and Jie Bai

Research on Irrigation System of Limited Water Supply for Soybean Crops in Shanxi Province . . . . . . . . . . . . . . . . . . . . . . . . . . . .

Lantao Ye, Yangren Wang, Qing Liu, and Sida Wang

Development and Application of Hyperspectral Remote Sensing . . . . . . . . . .

Huimin Xing, Haikuan Feng, Jingying Fu, Xingang Xu, and Guijun Yang

Study on Spatial Distribution Characters of Rubber Yield and Soil Nutrients in Guangba Farm of Hainai Province. . . . . . . . . . . . . . . . . . . . . . . . .

Bei Cui, Wenjiang Huang, Huichun Ye, and Qimin Cao

The Environment Intelligent Monitoring and Analysis for Enclosed Layer House with Four Overlap Tiers Cages in Winter. . . . . . . . . . . . .

Hualong Li, Miao Li, Junying Li, Kai Zhan, and Xianwang Liu

Research and Realization of Winter Wheat Yield Estimation Model Based on NDVI Index. . . . . . . . . . . . . . . . . . . . . . . . .

Zhichao Wu, Changchun Li, Haikuan Feng, Bo Xu, Guijun Yang, Zhenhai Li, Haojie Pei, and Mingxing Liu

Study on Vegetation Classification Based on Spectral Knowledge Base . . . . . Peng Liu, Jingcheng Zhang, Bin Wang, Xuexue Zhang, and Kaihua Wu 
Hyperspectral Estimation of Nitrogen Content in Winter Wheat Leaves

Based on Unmanned Aerial Vehicles. . . . . . . . . . . . . . . . . . .

Liu Mingxing, Li Changchun, Feng Haikuan, Pei Haojie, Li Zhenhai, Yang Fuqin, Yang Guijun, and Xu Shouzhi

Quantification of Root Anatomical Traits in $R G P$ Transgenic Maize Plants

Based on Micro-CT. . . . . . . . . . . . . . . . . . .

Xiaodi Pan, Liming Ma, Ying Zhang, Jinglu Wang, Jianjun Du, and Xinyu Guo

Effects of Exogenous Gamma-Aminobutyric Acid on Absorption and

Regulation of Ion in Wheat Under Salinity Stress . . . . . . . . . . . . . .

Xiaodong Wang, Hongtu Dong, Peichen Hou, Hang Zhou, Lulu He, and Cheng Wang

Development of Portable Dynamic Ion Flux Detecting Equipment. . . . . . . . .

Peichen Hou, Cheng Wang, Xiaodong Wang, Aixue Li, Peng Song,

Bin Luo, Ye Hu, and Liping Chen

Study on Intelligent Monitoring Technology for Composting

of Agricultural and Livestock Wastes . . . . . . . . . . . . . . . . . .

Hualong Li, Miao Li, Xuanjiang Yang, Zelin Hu, Zhirun Ma,

and Xianwang Liu

Optimization and Simulation of Fertilizer Guide Device Parameters Based

on EDEM Software. . . . . . . . . . . . . . . . . . . . . .

Hai Ding, Xiaofei An, Guangwei Wu, Liwei Li, and Qingzhen Zhu

Development of an Automated Guidance System for Tracked

Combine Harvester . . . . . . . . . . . . . . . . . . . . . . . . .

Fangming Zhang, Wenbin Wu, and Yunfei Zhu

Research on the Internet of Things Platform Design for Agricultural

Machinery Operation and Operation Management . . . . . . . . . . . . .

Qian Zhou, Jiandong Jiang, Zhangfeng Zhao, Jiang Zhong,

Bosong Pan, Xiao Jin, and Yuanfang Sun

Understanding the Consumer Satisfaction of the "Last-Mile" Delivery

of E-Business Services. . . . . . . . . . . . . . . . . . . . . .

Shujun Liu, Yan Li, Jingqi Huang, and Xin Zhao

The State of Motion Stereo About Plant Leaves Monitoring System Design and Simulation . . . . . . . . . . . . . . . . . . . . .

Jiangchuan Fan, Xinyu Guo, Chuanyu Wang, Xianju Lu, and Sheng Wu

Research on Agricultural Scientific and Technological Information

Dissemination System Based on Complex Network Technology . . . . . . . . . .

Hang Chen, Guifen Chen, Ying Zhang, and HongJun Gu 
An Illumination Invariant Maize Canopy Structure Parameters Analysis

Method Based on Hemispherical Photography . . . . . . . . . . . . .

Chuanyu Wang, Хinyu Guo, and Jianjun Du

Application of DBSCAN Algorithm in Precision Fertilization

Decision of Maize.

Yang Li, Guowei Wang, Yu Chen, Yang Jiao, Haijiao Yu, and Guogang Zhao

Location and Recognition Fruit Trees Based on Binocular Stereo Vision . . . . 460 Xueguan Zhao, Yuanyuan Gao, Songlin Wang, Xiu Wang, Pengfei Fan, and Qingcun Feng

The Realization of Pig Intelligent Feeding Equipment and Network Service Platform . . . . . . . . . . . . . . . . . . . . . .

Weihong Ma, Jinwei Fan, Chunjiang Zhao, and Huarui Wu

Improvement of Regional Spatial Interaction Based on Spatial Traffic

System Accessibility: A Case Study in Shandong Province, China

Yu Zhang, Shouzhi Xu, Fengguang Kang, and Shihua Yin

Research on the Key Techniques of Semantic Mining of Information Digest in the Field of Agricultural Major Crops Based on Deep Learning . . . . . . . . Hao G. J. M. Gong, Yunpeng Cui, and Ping Qian

COPS: A Real-Time Cross-Domain Object Part Segmentation System . . . . . . 508 Xueqing $\mathrm{He}$

Author Index 


\section{Contents - Part I}

Quinoa Traceable System Based on Internet of Things. . . . . . . . . . . . . 1

Guowei Wang, Yu Sun, Jing Chen, Yang Jiao, Chuanhong Zhang,

Haijiao Yu, Chan Lin, and Guogang Zhao

Research and Application of Safety Management for Virtual Desktop

in Colleges and Universities . . . . . . . . . . . . . . . . .

Haifeng Jia and Guifen Chen

Philosophical Principles of Data Discovery. . . . . . . . . . . . . . . .

Quan Wu, Min Liu, Juanying Sun, Weijie Jiao, Shuanghua Tao,

Xiaochen Li, Xue Han, and Lijuan Jia

Fast Analysis of Maize Kernel Plumpness Characteristics

Through Micro-CT Technology . . . . . . . . . . . . . . . . . . . . .

Meng Shao, Ying Zhang, Jianjun Du, Xiaodi Pan, Liming Ma,

Jinglu Wang, Dennis Böhmer, and Xinyu Guo

Automated Counting of Sex-Pheromone Attracted Insects

Using Trapped Images. . . . . . . . . . . . . . . . . . . . . .

Wenyong Li, Meixiang Chen, Ming Li, Chuanheng Sun, and Lin Wang

Study of Machine Learning Based Rice Breeding Decision Support

Methods and Technologies. . . . . . . . . . . . . . . . .

Yun-peng Cui, Jian Wang, Shi-hong Liu, En-ping Liu, and Hai-qing Liu

A Bayesian Network Model for Yellow Rust Forecasting in Winter Wheat. . . Xiaodong Yang, Chenwei Nie, Jingcheng Zhang, Haikuan Feng, and Guijun Yang

Soil Organic Carbon Prediction Using Vis-NIR Spectroscopy with a Large Dataset . . . . . . . . . . . . . . . . . . . . . . . . . .

Yang Shi, Rujing Wang, and Yubing Wang

Research on High Resolution Remote Sensing Image Classification

Based on Convolution Neural Network . . . . . . . . . . . . . . . . .

Wenwen Gong, Zhuqing Wang, Yong Liang, Xin Fan, and Junmeng Hao

Computer Vision and Feeding Behavior Based Intelligent Feeding

Controller for Fish in Aquaculture. . . . . . . . . . . . . . . . . . . .

Chao Zhou, Kai Lin, Daming Xu, Chuanheng Sun, Lan Chen, Song Zhang, and Qiang Guo 
Multi-scale 3D Data Acquisition of Maize . . . . . . . . . . . . 108 Weiliang Wen, Xinyu Guo, Xianju Lu, Yongjian Wang, and Zetao Yu

Remote Sensing Monitoring of Drought Based on Landsat8 and NDVI-Ts

Characteristic Space Method. . . . . . . . . . . . . . . . . . . . Shouzhen Liang, Tao Liu, Zhen Chen, Xueyan Sui, Xuehui Hou, Meng Wang, and Huimin Yao

Curve Fitting Derivative Method and Its Application in Mouse Growth . . . . . Zhihua Li and Xin Zhao

Spatial Structure Change Analysis of Cultivated Soil Nutrients in Urban Fringe of North China . . . . . . . . . . . . . . . . . Shiwei Dong, Yuchun Pan, and Bingbo Gao

Evaluation and Mapping of Rice Flood Damage Using Domestic Remotely Sensed Data in China . . . . . . . . . . . . . . . . . . Huifang Wang, Xiaoyi Fang, Wei Guo, Yonghong Liu, Qingzu Luan, Shuo Zhang, and Yanhu Gao

Estimating Leaf Carotenoid Concentration of Ginger in Different Layers Based on Discrete Wavelet Transform Algorithm . . . . . . . . . . . . . . Qinhong Liao

Ecological Footprint Model of Cultivated Land Based on Ecosystem

Services in Beijing ........................

Hui Guo, Di Wu, Lei Fa, Shunxiang Pei, Xuebing Xin, Shumin Ma, Sha Wu, and Shiwei Dong

Numerical Simulation of the Effects of Design Parameters on the Performance of Tractor Powered Flail Choppers . . . . . . . . . . . . . Zhiqiang Zhang, Hongwen Li, Allen D. McHugh, Jin He, Qingjie Wang, Caiyun Lu, Wenzheng Liu, and Sun Nina

Design and Implementation of Water Spectrum Observation System for Aquaculture Pond . . . . . . . . . . . . . . . . . . . . . . Yinchi Ma, Wen Ding, Yonghua Qu, and Xiande Zhao

Chlorophyll Fluorescence Measurement: A New Method to Test the Effect of Two Adjuvants on the Efficacy of Topramezone on Weeds . . . . . . . . . 206 Jinwei Zhang, Ortrud Jäck, Alexander Menegat, Gen Li, and Xiu Wang

Wheat Growth Process 3D Visualization Research Based on Growth Model. . . Hailong Liu, Shuqin Li, Yeping Zhu, Shengping Liu, and Shijuan Li

Research on Information Integration Method of Agricultural Products Producing and Managing Based on Knowledge Graph. . . . . . . . . . . . . . . Xiang Sun, Huarui Wu, Peng Hao, and Qingxue Li 
A 3D Canopy Reconstruction and Phenotype Analysis Method for Wheat ... Boxiang Xiao, Sheng Wu, Xinyu Guo, and Weiliang Wen

Detection of Young Green Apples in Orchard Environment Using Adaptive Ratio Chromatic Aberration and HOG-SVM. . . . . . . . . . . . . . . . .

Xia Xue, Zhou Guomin, Qiu Yun, Li Zhuang, Wang Jian, Hu Lin, Fan Jingchao, and Guo Xiuming

Detection of Overlapped Apples in Orchard Scene Using Improved K-means and Distance Least Square . . . . . . . . . . . . . . . . . .

Xia Xue, Zhou Guomin, Qiu Yun, Li Zhuang, Wang Jian, Hu Lin, Fan Jingchao, and Guo Xiuming

Application of Growth Curve in Agricultural Scientific Research . . . . . . . . Zeng-hui Wang, Yan-jun Zhao, Yang Liu, and Dong-yan Huang

The Study of the Work Parameters of the Corn Harvester Cutter. . . . . . . . . . Zeng-hui Wang, Yan-jun Zhao, Yang Liu, and Dong-yan Huang

Test Device of Soil Outline and Compactness Distribution on Seedbed Based on Sensors . . . . . . . . . . . . . . . . . . . . . . .

Caiyun Lu, Liwei Li, Zhijun Meng, Xiu Wang, and Qingjie Wang

Study the Spatial-Temporal Variation of Wheat Growth Under Different Site-Specific Nitrogen Fertilization Approaches. . . . . . . . . . . . . . Bei Cui, Wenjiang Huang, Xiaoyu Song, Huichun Ye, and Yingying Dong

Classification Method Research of Fresh Agaricus Bisporus Based on Image Processing . . . . . . . . . . . . . . . . . .

Fengyun Wang, Jiye Zheng, Lei Wang, Wenjie Feng, and Luyan Niu

A Study About Searching Behavior of Scientific Data User Based on Educational Background and Retrieval Capability. . . . . . . . . . . . .

Guilan Zhang, Jian Wang, Guomin Zhou, Jianping Liu, Fei Gao, and Caoyuan Wei

A Review on the Soil Moisture Prediction Model and Its Application in the Information System . . . . . . . . . . . . . . . . . . .

Wengang Zheng, Lili Zhangzhong, Xin Zhang, Caiyuan Wang, Shirui Zhang, Shijun Sun, and Hongfei Niu

Application of Image Segmentation Technology in Crop Disease Detection

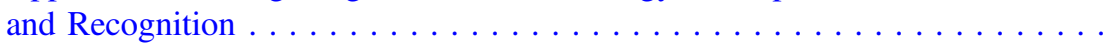

Leilei Deng, Zhenghao Wang, and Hui Zhou 
Research and Realization on the Performance Testing Tool of Web Application. . . . . . . . . . . . . . . . . . . . . .

Huarui Wu and Huaji Zhu

Operation Area Measurement Based on Trajectories

of Agricultural Machinery . . . . . . . . . . . . . . . . . . . . . .

Chang Ren, Yanwei Yuan, Liwei Yang, Junning Zhang, Yangchun Liu,

Chengxu Lv, and Bo Zhao

Analysis of Influential Factors of Social Satisfaction in Food Industry . . . . . .

Zhiyu Lai, Xinyao Zhu, Chenyue Jin, Mingxin Li, and Yan Qi

Abnormal Identification of Swine Flu Clinical Characteristics Based

on Body Temperature and Behavior . . . . . . . . . . . . . . .

404

Duo Wang, Ying Xu, Qifeng Liu, Yue Lou, Chaorong Luo,

and Changji Wen

Hierarchical Denoising Method of Crop 3D Point Cloud Based

on Multi-view Image Reconstruction .

Lei Chen, Yuan Yuan, and Shide Song

Non-invasive Edge Detection of Leaves Based on Order Morphology . . . . . . Yanlei Xu, Qi Zhang, Chenxiao Li, Xindong Wang, and Xiaotian Meng

Development and Test of GNSS/IMU-Based Speed Measurement Device for Agricultural Machinery. . . . . . . . . . . . . . . . . . . . . . . . .

Weiqiang Fu, Shupeng Hu, Changhai Luo, You Li, Shuxia Guo, and Junxiong Zhang

Farmland Weed Species Identification Based on Computer Vision . . . . . . . . Shengping Liu, Junchan Wang, Liu Tao, Zhemin Li, Chengming Sun, and Xiaochun Zhong

Remote Monitoring and Control System for Aquarium Based on Mobile

Communication Platform . . . . . . . . . . . . . . . . . . .

Wen Ding, Yinchi Ma, and Jinjing Zhang

Effects of the Factors on Maize Yield Under Drip Irrigation Under Film . . . . 468 Hongzheng Shen, Yangren Wang, Xinrui Fan, Hao Wang, and Yonglin $\mathrm{Li}$

An Amperometric Glucose Microbiosensor for Real-Time Measurements

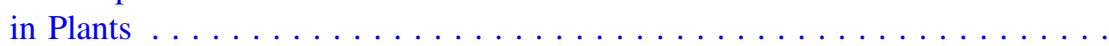

Ye Hu, Cheng Wang, and Aixue Li

Field Information Recommendation Based on Context-Aware and Collaborative Filtering Algorithm . . . . . . . . . . . . . . .

Zhili Chen, Chunjiang Zhao, and Huarui Wu 
Research on the Current Situation and Development of Intelligent Precision

Fertilizer Technology. . . . . . . . . . . . . . . . . . . . .

Wu Yan and Wang Fan

The Design and Analysis of Self-Balancing Adjustment Implement

Leveling Control System . . . . . . . . . . . . . . . . . .

Qingfeng Yang, Yehua Shang, Siyu Liu, Fujie Zhang, Yue Cong,

Weiqiang Fu, Rui Pan, and Chunjiang Zhao

Research on Automatic Steering Control System of Full Hydraulic

Steering Tractor . . . . . . . . . . . . . . . . . . .

Shupeng Hu, Weiqiang Fu, You Li, Yue Cong, Yehua Shang,

and Zhijun Meng

Design of Farmland Information Acquisition System Based on LoRa

Wireless Sensor Network . . . . . . . . . . . . . . . . . . . . .

Qiulan Wu, Chuanqi Zhao, Yong Liang, Dalei Zhang, and Junmeng Hao

Multi-sensor Array Based Fire Monitor for Cotton Pile .

Chenrui Bai, Junning Zhang, Chengxu Lv, Liguo Wei, Liming Zhou, and Bo Zhao 